\title{
Covariant Spectator Theory: Foundations and Applications
}

\section{A mini-review of the covariant spectator theory}

\author{
Alfred Stadler · Franz Gross
}

Received: date / Accepted: date

\begin{abstract}
We provide a short overview of the Covariant Spectator Theory and its applications. The basic ideas are introduced through the example of a $\phi^{4}$-type theory. Highprecision models of the two-nucleon interaction are presented and the results of their use in calculations of properties of the two- and three-nucleon systems are discussed. A short summary of applications of this framework to other few-body systems is also presented.
\end{abstract}

Keywords Relativistic few-body systems · Nuclear interaction

\section{Introduction}

The purpose of the Covariant Spectator Theory (CST) [1,2] is to provide a relativistic framework for the description of few-body systems in general, and for systems containing a small number of nucleons in particular.

To the best of our knowledge, the laws of nature are constrained by the principle of relativity. In the absence of significant space-time curvature this means that the physical laws should have the same form in all inertial reference frames, i.e., they should be covariant.

"Relativistic Description of Two- and Three-Body Systems in Nuclear Physics", ECT*, October 19-13 2009

\footnotetext{
A. Stadler

Departamento de Física da Universidade de Évora, 7000-671 Évora, Portugal

and Centro de Física Nuclear da Universidade de Lisboa, 1649-003

Lisboa, Portugal

E-mail: stadler@uevora.pt

F. Gross

Thomas Jefferson National Accelerator Facility, Newport News, VA 23606, USA

and College of William and Mary, Williamsburg, VA 23187, USA

E-mail: gross@jlab.org
}

Nonrelativistic Quantum Mechanics (NRQM) is clearly not covariant, but has been very successful in describing many few-nucleon phenomena at low energies (roughly, below the pion-production threshold). From typical average velocities of nucleons in nuclei one can estimate that relativistic corrections to their kinetic energy should not exceed a few percent. Why, then, should one make the effort of constructing a substantially more complicated relativistic theory?

Here are a few reasons:

(i) Few-nucleon physics has entered an era of high-precision experiments. Even if relativity should turn out to lead to only small differences compared to nonrelativistic theory, these differences might be experimentally detectable and therefore significant.

(ii) There are some discrepancies between measured observables and theoretical calculations based on NRQM with the most accurate nucleon-nucleon $(N N)$ potentials that remain still unexplained. Examples are the well-known $A_{y^{-}}$ puzzle in $N d$ scattering, and the underprediction of the threenucleon $(3 N)$ binding energy (usually fixed by the introduction of three-body forces) and the deuteron quadrupole moment. It is important to know if relativity can explain these discrepancies, at least in part.

(iii) Experience with the Dirac equation has taught us that observables that depend on the nuclear spin can be especially well described by a relativistic treatment, which can sometimes unify phenomena that might appear to be otherwise unrelated when studied from a nonrelativistic point of view.

(iv) Eventually, relativity becomes unavoidable: for example, when the structure of light nuclei is investigated by means of scattering with hadronic or electromagnetic probes, the transferred momentum can become so large that NRQM is no longer sufficient to describe the high-velocity final nuclear state. The validity of adding relativistic corrections per- 


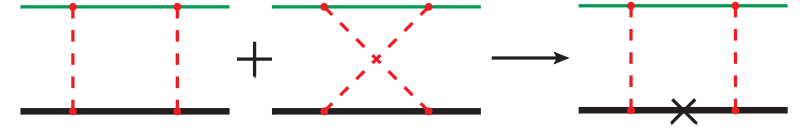

Fig. 1 In $\phi^{3}$-type theories, as the mass of the heavy particle goes to infinity, the sum of box and crossed-box diagrams becomes equal to the box diagram with the heavy particle on mass shell (indicated by a cross on the line).

turbatively to calculations with NRQM can only be tested when it can be compared to truly covariant calculations.

(v) Finally, the study of relativistic equations is interesting from the theoretical point of view. There are several ways to extend NRQM to include the requirements of special relativity. They can be divided into two groups: formalisms in which the number of particles is fixed, referred to collectively as Relativistic Hamiltonian Dynamics, and others based on Relativistic Quantum Field Theory, in which the number of particles is not fixed. There are many interesting conceptional questions about how the various forms of relativistic theories within each group are related to each other, and perhaps even more about the relations between the two groups.

\section{Fundamental ideas of CST}

The basic idea of the CST is to reorganize the manifestly covariant Bethe-Salpeter (BS) equation with its complete kernel to an equivalent form, in which a different propagator and an accordingly modified kernel is used. The new propagator is chosen such that, when the kernel of the reorganized equation is truncated, the dimension of the integration over intermediate momenta reduces from four to three, while maintaining the covariance of the equation.

There are many ways to rearrange the BS equation leading to a dimensional reduction. The resulting equations are called "quasi-potential equations." While the CST belongs in this category, it has properties not all of the other quasipotential theories possess. For instance, it satisfies the property of cluster-separability, without which a calculated twobody scattering amplitude could not be used consistently in the kernel of a three-body equation. It also has the one-body limit: when one particle is a massive scalar, the two body equation reduces to a relativistic one body equation for the light particle moving in an effective potential created by the massive particle.

The defining feature of the CST is that it places in intermediate states all particles but one on their mass shells [1, 2]. This choice was originally motivated by an observed cancellation between two-body box and crossed-box diagrams in scalar theories of $\phi^{3}$-type, where two heavier particles with unequal masses exchange a third lighter one. As illustrated in Fig. 1, when the mass of the heaviest particle in-

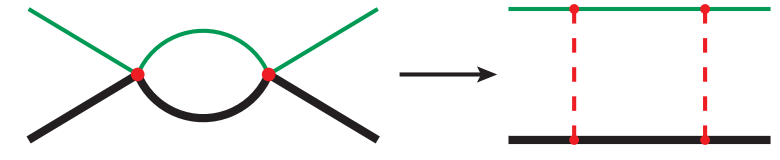

Fig. 2 The relation of the s-channel bubble diagram to the box diagram when the four-point vertex is "opened" and transformed into a boson exchange.

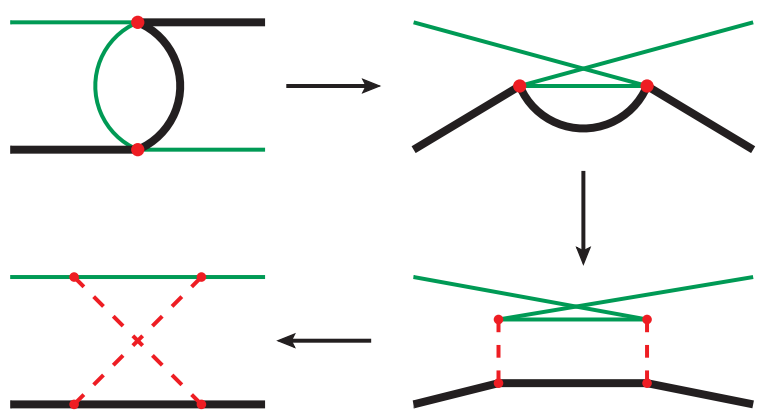

Fig. 3 The relation of the u-channel bubble diagram to the crossed box when the four-point vertex is "opened" and transformed into a boson exchange.

creases, its positive-energy pole dominates the sum of box and crossed box diagrams, while the remainder tends to cancel. In fact, this cancellation occurs in diagrams of all orders, and if the heavy mass goes to infinity, the exact solution is obtained from a one boson exchange (OBE) interaction with the heavy particle on mass shell in all intermediate states.

It was originally believed that the exchanged particle had to be light for these cancellations to occur. However, we show here that the same kind of cancellations appear also in theories of $\phi^{4}$-type, which can be interpreted as limits of $\phi^{3}$-type theories with infinite exchanged mass, as illustrated in Figs. 2 and 3. When the four-point vertex is "opened" to represent a one-boson exchange, the s-channel diagram becomes the box diagram, and the u-channel-after some additional deformation without changing its topology—becomes the crossed-box diagram.

Consider a $\phi^{4}$-type theory with two scalar fields, one neutral with mass $M$ called $\psi$, and one charged with mass $m<M$ called $\phi$, interacting through $\mathscr{L}_{I}=-\lambda \phi^{\dagger} \phi \psi^{2}$. The two lowest-order diagrams that contribute to the scattering amplitude are

$\mathscr{M}_{s}=i \lambda^{2} \int \frac{d^{4} k}{(2 \pi)^{4}}\left[\frac{1}{m^{2}-k^{2}-i \varepsilon}\right] \frac{1}{M^{2}-(P-k)^{2}-i \varepsilon}$

for the s-channel, and

$\mathscr{M}_{u}=i \lambda^{2} \int \frac{d^{4} k}{(2 \pi)^{4}}\left[\frac{1}{m^{2}-k^{2}-i \varepsilon}\right] \frac{1}{M^{2}-(X+k)^{2}-i \varepsilon}$

for the u-channel, where $X \equiv P-p^{\prime}-p$, and the four-momenta $p, p^{\prime}$, and $k$ are defined in Figs. 4 and 5. 


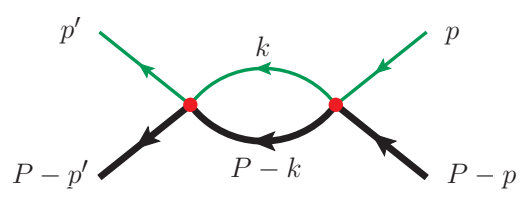

Fig. 4 The s-channel bubble diagram in a $\phi^{4}$-type theory.

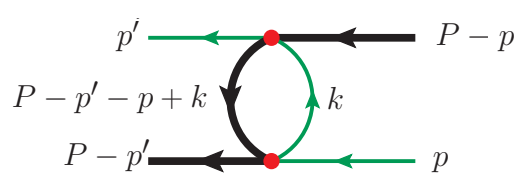

Fig. 5 The u-channel bubble diagram in the $\phi^{4}$-type theory. In the text we substitute $X=P-p^{\prime}-p$.

We evaluate these diagrams in the two-body rest frame, where the total momentum is $P=(W, 0)$ with $W$ the total energy. Assuming that the particles in the initial and final states are on mass shell, so that their energies are $E_{p}=\sqrt{M^{2}+p^{2}}$ and $e_{p}=\sqrt{m^{2}+\boldsymbol{p}^{2}}$, we have $W=E_{p}+e_{p}=E_{p^{\prime}}+e_{p^{\prime}}$, and therefore $|\boldsymbol{p}|=\left|\boldsymbol{p}^{\prime}\right|$.

After determining the positions of the poles in the complex $k_{0}$ plane, the integral over $k_{0}$ can be performed, giving

$$
\begin{aligned}
\mathscr{M}_{s}=-\lambda^{2} \int \frac{d^{3} k}{(2 \pi)^{3}}\{ & \frac{1}{2 E_{k}\left[e_{k}^{2}-\left(E_{p}+e_{p}-E_{k}\right)^{2}\right]} \\
& \left.+\frac{1}{2 e_{k}\left[E_{k}^{2}-\left(E_{p}+e_{p}+e_{k}\right)^{2}\right]}\right\} .
\end{aligned}
$$

Keeping track of the origin of the various terms, the first term in Eq. (3) comes from the positive-energy pole of the heavy particle while the second comes from the negativeenergy pole of the light particle. For the u-channel we get

$$
\begin{aligned}
\mathscr{M}_{u}=-\lambda^{2} \int \frac{d^{3} k}{(2 \pi)^{3}}\{ & \frac{1}{2 E_{x}\left[e_{k}^{2}-\left(E_{x}+E_{p}-e_{p}\right)^{2}\right]} \\
& \left.+\frac{1}{2 e_{k}\left[E_{x}^{2}-\left(e_{k}+e_{p}-E_{p}\right)^{2}\right]}\right\},
\end{aligned}
$$

where $\boldsymbol{x}=\boldsymbol{k}-\boldsymbol{p}-\boldsymbol{p}^{\prime}$. Both terms in Eq. (4) come from positive-energy poles, the first from the heavy and the second from the light particle.

The integrands inside the braces in Eqs. (3) and (4) can be rewritten as

$$
\begin{array}{r}
I_{s}=\frac{1}{4 e_{k} E_{k}}\left\{\frac{1}{E_{k}-E_{p}+e_{k}-e_{p}}+\frac{1}{-E_{k}+E_{p}+e_{k}+e_{p}}\right. \\
\left.+\frac{1}{E_{k}-E_{p}-e_{k}-e_{p}}+\frac{1}{E_{k}+E_{p}+e_{k}+e_{p}}\right\}
\end{array}
$$

and

$$
\begin{array}{r}
I_{u}=\frac{1}{4 e_{k} E_{x}}\left\{\frac{1}{-E_{x}-E_{p}+e_{k}+e_{p}}+\frac{1}{E_{x}+E_{p}+e_{k}-e_{p}}\right. \\
\left.+\frac{1}{E_{x}+E_{p}-e_{k}-e_{p}}+\frac{1}{E_{x}-E_{p}+e_{k}+e_{p}}\right\} .
\end{array}
$$

For large values of $M$ we can approximate $E_{k} \simeq M+\frac{k^{2}}{2 M}$ (the same applies to $E_{p}$ and $E_{x}$ ). After factoring out $M$ we obtain

$$
\begin{aligned}
& I_{s}+I_{u}=\frac{1}{4 M e_{k}}\left\{\frac{1}{e_{k}-e_{p}+\frac{k^{2}-p^{2}}{2 M}+\frac{k^{2}\left(e_{k}-e_{p}\right)}{2 M^{2}}}\right. \\
& +\frac{1}{e_{k}+e_{p}-\frac{k^{2}-p^{2}}{2 M}+\frac{k^{2}\left(e_{k}+e_{p}\right)}{2 M^{2}}} \\
& +\frac{1}{-e_{k}-e_{p}+\frac{k^{2}-p^{2}}{2 M}-\frac{k^{2}\left(e_{k}+e_{p}\right)}{2 M^{2}}} \\
& +\frac{1}{2 M+e_{k}+e_{p}+\frac{3 k^{2}+p^{2}}{2 M}+\frac{k^{2}\left(e_{k}+e_{p}\right)}{2 M^{2}}} \\
& +\frac{1}{-2 M+e_{k}+e_{p}-\frac{3 x^{2}+p^{2}}{2 M}+\frac{x^{2}\left(e_{k}+e_{p}\right)}{2 M^{2}}} \\
& +\frac{1}{2 M+e_{k}-e_{p}+\frac{3 x^{2}+p^{2}}{2 M}+\frac{x^{2}\left(e_{k}-e_{p}\right)}{2 M^{2}}} \\
& +\frac{1}{2 M-e_{k}-e_{p}+\frac{3 \boldsymbol{x}^{2}+\boldsymbol{p}^{2}}{2 M}-\frac{\boldsymbol{x}^{2}\left(e_{k}+e_{p}\right)}{2 M^{2}}} \\
& \left.+\frac{1}{e_{k}+e_{p}+\frac{\boldsymbol{x}^{2}-\boldsymbol{p}^{2}}{2 M}+\frac{\boldsymbol{x}^{2}\left(e_{k}+e_{p}\right)}{2 M^{2}}}\right\} \text {. }
\end{aligned}
$$

The first four terms come from the s-channel, the second four from the u-channel. The fifth and seventh term cancel exactly, and as $M$ becomes very large, the fourth and sixth terms can be neglected, as well as the terms proportional to $1 / M$ or $1 / M^{2}$ in the denominators of the other terms. This leaves only the first three and the last term, leaving finally

$$
\begin{aligned}
I_{s}+I_{u} & \rightarrow \frac{1}{4 M e_{k}}\left\{\frac{1}{e_{k}-e_{p}}+\frac{1}{e_{k}+e_{p}}+\frac{1}{-e_{k}-e_{p}}+\frac{1}{e_{k}+e_{p}}\right\} \\
& =\frac{1}{4 M e_{k}}\left\{\frac{1}{e_{k}-e_{p}}+\frac{1}{e_{k}+e_{p}}\right\} .
\end{aligned}
$$

where the second line shows the result with the last two terms cancelled. The two terms remaining come only from the positive energy pole in the s-channel.

There are two important conclusions we can draw from this result:

(1) The u-channel amplitude is not negligible, since the fourth term in the first line of Eq. (8) is of the same order as the other three terms, which originate from the s-channel.

(2) The u-channel amplitude cancels part of the s-channel amplitude, leaving only the contribution from the positiveenergy pole of the heavy particle. This means that the contribution of the positive-energy pole of the heavy particle to the s-channel amplitude turns into the exact result as its mass goes to infinity.

In other words: the exact result for the sum of the s- and u-channel diagrams is obtained only from the part of the schannel diagram where the heavy particle is placed on its 


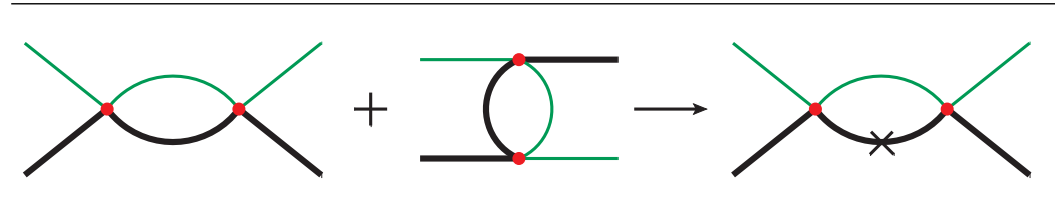

Fig. 6 Illustration of the cancellation theorem for $\phi^{4}$ type theories. positive-energy mass-shell, graphically represented in Fig. 6. Taking into consideration the relation between the $\phi^{3}$ type and $\phi^{4}$-type theories illustrated in Figs. 2 and 3, the cancellation of Fig. 6 is closely related to the one of Fig. 1.

The special choice for the intermediate-state propagators of CST of placing particles on mass shell is motivated by the kind of cancellations just described, for which the lowestorder kernel gives the exact result. However, in the general case the complete kernel of the CST equation contains an infinite number of irreducible diagrams, which makes it equivalent to the BS equation.

Note that when the kernel is truncated, the exact equivalence to the full BS equation is lost, and that the cancellations are not complete for finite (or even equal) mass particles. They are also know to be incomplete when charged particles are exchanged. However, this would only be an issue were one to attempt to find an exact solution of a field theory for a given Lagrangian. Instead, the equations of CST can be taken as the starting point for a description of few-body systems that is in part phenomenological; they are based on certain physical mechanisms, such as one-boson exchange (OBE), but they have phenomenological aspects in the way integrations are regularized, and in the use of parameters, some of which have to be determined from fits to experimental data.

\section{Covariant Spectator Theory of two- and three-nucleon systems}

\subsection{Two-nucleon equation}

A good understanding of the interaction between two nucleons is essential for the study of nuclear structure and nuclear reactions. The construction of a CST kernel for $N N$ scattering which reproduces the deuteron properties and the $N N$ scattering observables is therefore of high importance.

First we introduce the CST equation for two-body scattering. Its general form is

$M=V-V G M$,

where $V$ is the irreducible kernel (which we also refer to as the "potential"), and $G$ is the intermediate state propagator.

The specific form of the CST equation for the $N N$ scattering amplitude $M$, with particle 1 on-shell in both the ini- tial and final state, is [3]

$$
\begin{aligned}
& M_{12}\left(p, p^{\prime} ; P\right)=\bar{V}_{12}\left(p, p^{\prime} ; P\right) \\
& -\int \frac{d^{3} k}{(2 \pi)^{3}} \frac{m}{E_{k}} \bar{V}_{12}(p, k ; P) G_{2}(k, P) M_{12}\left(k, p^{\prime} ; P\right),
\end{aligned}
$$

where $P$ is the conserved total four-momentum, and $p, p^{\prime}$, and $k$ are relative four-momenta related to the momenta of particles 1 and 2 by $p_{1}=\frac{1}{2} P+p, p_{2}=\frac{1}{2} P-p$, and

$$
\begin{aligned}
M_{12}\left(p, p^{\prime} ; P\right) & \equiv M_{\lambda \lambda^{\prime}, \beta \beta^{\prime}}\left(p, p^{\prime} ; P\right) \\
& =\bar{u}_{\alpha}(\mathbf{p}, \lambda) \mathscr{M}_{\alpha \alpha^{\prime} ; \beta \beta^{\prime}}\left(p, p^{\prime} ; P\right) u_{\alpha^{\prime}}\left(\mathbf{p}^{\prime}, \lambda^{\prime}\right)
\end{aligned}
$$

is the matrix element of the Feynman scattering amplitude $\mathscr{M}$ between positive energy Dirac spinors of particle 1 . The indices 1 and 2 refer collectively to the two helicity or Dirac indices of particle 1 , either $\left\{\lambda \lambda^{\prime}\right\}$ or $\left\{\alpha \alpha^{\prime}\right\}$, and particle 2 , $\left\{\beta \beta^{\prime}\right\}$. We use the notation that the replacement of a Dirac index $\left(\alpha_{i}, \beta_{i}, \ldots\right)$ by a helicity index $\left(\lambda_{i}\right)$ always indicates a corresponding contraction with a positive-energy helicity spinor. A closed set of equations is obtained when particle 2 is placed on mass shell in the initial state.

The propagator for the off-shell particle 2 is dressed by the off-shell nucleon form factor $h$

$G_{2}(k, P) \equiv G_{\beta \beta^{\prime}}\left(k_{2}\right)=\frac{\left(m+k_{2}\right)_{\beta \beta^{\prime}}}{m^{2}-k_{2}^{2}-i \varepsilon} h^{2}\left(k_{2}\right)$

with $k_{2}=P-k_{1}, k_{1}^{2}=m^{2}$, and $h\left(k_{2}\right)$, which can be related to the self-energy of the off-shell nucleon, is normalized to unity when $k_{2}^{2}=m^{2}$. In Ref. [4] we used

$h(p)=\left[\frac{\left(\Lambda_{N}^{2}-m^{2}\right)^{2}}{\left(\Lambda_{N}^{2}-m^{2}\right)^{2}+\left(m^{2}-p^{2}\right)^{2}}\right]^{2}$,

where $\Lambda_{N}$ is an adjustable cutoff parameter.

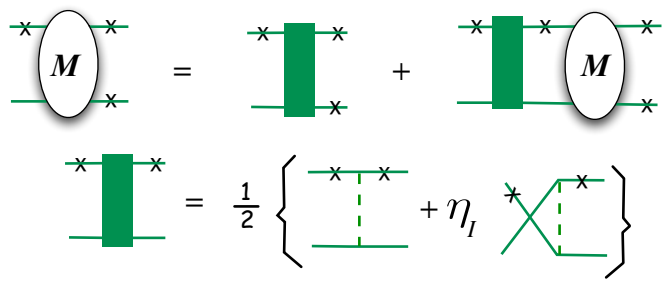

Fig. 7 Top line: diagramatic representation of the Covariant Spectator equation (10) with particle 1 on-shell (the on-shell particle is labeled with $\mathrm{a} \times)$. Second line: diagrammatic representation of the definition of the antisymmetrized kernel (14), with $\eta_{I}=(-)^{I}$. 


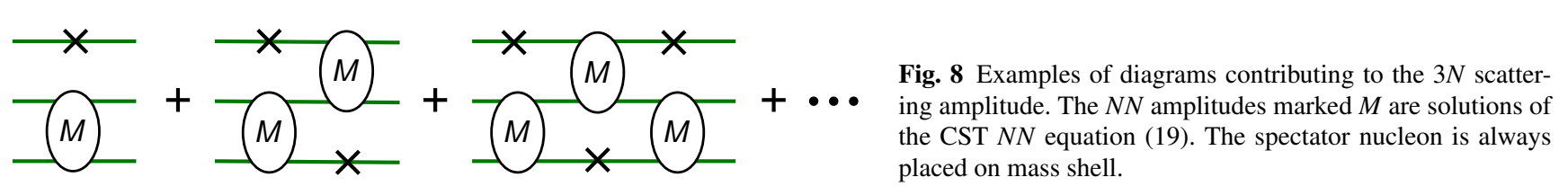

The Dirac form of the explicitly antisymmetrized covariant kernel $\bar{V}$ is

$$
\begin{aligned}
& \bar{V}_{\alpha \alpha^{\prime} ; \beta \beta^{\prime}}(p, k ; P) \\
& =\frac{1}{2}\left[V_{\alpha \alpha^{\prime} ; \beta \beta^{\prime}}(p, k ; P)+(-)^{I} V_{\beta \alpha^{\prime} ; \alpha \beta^{\prime}}(-p, k ; P)\right],
\end{aligned}
$$

where the isospin indices have been suppressed, so that the factor of $(-)^{I}$ (with $I=0$ or 1 the isospin of the $N N$ state) insures that the remaining amplitude has the symmetry $(-)^{I}$ under particle interchange $\left\{p_{1}, \alpha\right\} \leftrightarrow\left\{p_{2}, \beta\right\}$, as required by the generalized Pauli principle. This symmetry insures that identical results emerge if a different particle is chosen to be on-shell in either the initial or final state.

The bare propagator of an off-shell particle with fourmomentum $k$ can be decomposed into positive and negative energy contributions

$$
\begin{aligned}
& \frac{(m+k)_{\beta \beta^{\prime}}}{m^{2}-k^{2}-i \varepsilon}= \\
& \quad \frac{m}{E_{k}} \sum_{\lambda}\left\{\frac{u_{\beta}(\boldsymbol{k}, \lambda) \bar{u}_{\beta^{\prime}}(\boldsymbol{k}, \lambda)}{E_{k}-k^{0}}-\frac{v_{\beta}(-\boldsymbol{k}, \lambda) \bar{v}_{\beta^{\prime}}(-\boldsymbol{k}, \lambda)}{E_{k}+k^{0}}\right\} .
\end{aligned}
$$

Accordingly, the CST equations can be separated into positive- and negative-energy (also called " $\rho$-spin"- ) channels, which is useful for their numerical solution. However, the decomposition (15) is frame-dependent and thus breaks manifest covariance. This does not mean that the $\rho$-spin separated equations are no longer covariant, only that their covariance is no longer explicit as it is in the manifestly covariant form.

Negative-energy states are related to the "Z-graphs" of time-ordered perturbation theory. In this sense, the solutions of Eq. (10) automatically include Z-graphs to all orders. This is useful to keep in mind when CST is compared to other theories in which relativistic corrections are added perturbatively to nonrelativistic calculations.

\subsection{Three-nucleon equation}

The CST of $3 N$ scattering was formulated for the first time in Ref. [2]. Assuming only two-body interactions, the main idea is to place spectators always on mass shell. In fact, the name "spectator theory" derives from this principle and is best illustrated in the three-body problem. If this is done consistently, in any intermediate state there are always two nucleons on mass shell, and one off mass shell, as illustrated in Fig. 8. This has the important consequence that the energy-component in all loop integrations is constrained by total energy conservation and two mass-shell conditions, such that all loop integrations are three-dimensional.

Furthermore, in the case of short-range interactions, cluster separability requires that when any one particle is far removed from the remaining pair, the system has to separate into a free particle and an independent two-particle system with internal interactions only. As long as the particles are close together, each of them is sometimes a spectator and sometimes participates in a pair interaction. However, when one particle's distance to the others increases, its interaction with them eventually vanishes completely (only the first diagram in Fig. 8 remains). It becomes a permanent spectator, and therefore in CST it is automatically always on mass shell. This is of course exactly what is required for a free particle.

In Ref. [5] the CST equations for the $3 N$ bound state were formulated in a way suitable for a practical solution. Starting from Faddeev-type integral equations for the $3 \mathrm{~N}$ scattering amplitude $\mathscr{M}_{3 N}$, one looks for a pole at the subthreshold bound-state energy $M_{t}$, i.e., where it can be written as

$\mathscr{M}_{3 N}=-\frac{\Gamma \bar{\Gamma}}{M_{t}^{2}-P^{2}}+\mathscr{R}$.

Here, $\mathscr{R}$ is the remaining non-pole contribution, and $P=$ $k_{1}+k_{2}+k_{3}$ is the total four-momentum of the $3 N$ system. One obtains a homogeneous equation for the vertex function $\Gamma$ of the bound state,

$$
\begin{aligned}
\Gamma_{\lambda_{1} \lambda_{2} \alpha}\left(k_{1}, k_{2} ; P\right) & =2 \int \frac{d^{3} k_{2}^{\prime}}{(2 \pi)^{3}} \frac{m}{E_{k_{2}^{\prime}}} \sum_{\lambda_{2}^{\prime}} M_{\lambda_{2} \alpha, \lambda_{2}^{\prime} \alpha^{\prime}}\left(k_{2}, k_{2}^{\prime} ; P_{23}\right) \\
\times & \mathscr{P}_{12} G_{\alpha^{\prime} \beta}\left(P_{23}-k_{2}^{\prime}\right) \Gamma_{\lambda_{1} \lambda_{2}^{\prime} \beta}\left(k_{1}, k_{2}^{\prime} ; P\right),
\end{aligned}
$$

where the permutation operator $\mathscr{P}_{12}$ interchanges particles 1 and 2 (see also Fig. 9). The particular vertex function in Eq. (17) is for the case that particle 1 was the spectator during the "last" interaction, and particle 2 is the second onshell particle. For identical particles, all other vertex functions are related to it by exchange symmetry.

As in Eq. (11), we use a shorthand for the contraction of Dirac indices with nucleon helicity spinors with helicity $\lambda_{i}$,

$$
\begin{aligned}
& \Gamma_{\lambda_{1} \lambda_{2} \alpha^{\prime}}\left(k_{1}, k_{2} ; P_{t}\right) \equiv \\
& \bar{u}_{\alpha_{1}}\left(k_{1}, \lambda_{1}\right) \bar{u}_{\alpha_{2}}\left(k_{2}, \lambda_{2}\right) \Gamma_{\alpha_{1} \alpha_{2} \alpha^{\prime}}\left(k_{1}, k_{2} ; P_{t}\right),
\end{aligned}
$$




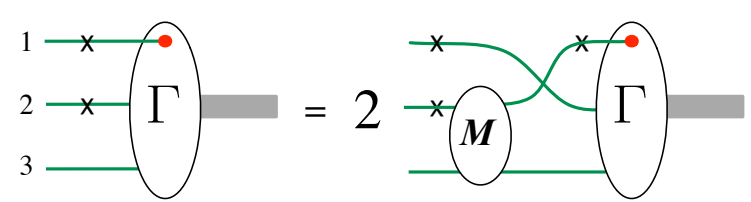

Fig. 9 Diagramatic representation of the Covariant Spectator equation for the three-body bound state vertex function $\Gamma$ with particles 1 and 2 on-shell (labeled with a $\times$ ). Here particle 1 is the spectator to the last two-body interaction between particles 2 and 3, described by the scattering amplitude $M$ with particle 3 off-shell.

and $M_{\lambda_{2} \alpha, \lambda_{2}^{\prime} \alpha^{\prime}}\left(k_{2}, k_{2}^{\prime} ; P_{23}\right)$ is the scattering amplitude of nucleons 2 and 3 with total pair momentum $P_{23}$. It satisfies the CST two-body equation

$$
\begin{aligned}
& M_{\lambda_{2} \alpha, \lambda_{2}^{\prime} \alpha^{\prime}}\left(k_{2}, k_{2}^{\prime} ; P_{23}\right)=\bar{V}_{\lambda_{2} \alpha, \lambda_{2}^{\prime} \alpha^{\prime}}\left(k_{2}, k_{2}^{\prime} ; P_{23}\right) \\
& -\int \frac{d^{3} k_{2}^{\prime \prime}}{(2 \pi)^{3}} \frac{m}{E_{k_{2}^{\prime \prime}}} \sum_{\lambda_{2}^{\prime \prime}} \bar{V}_{\lambda_{2} \alpha, \lambda_{2}^{\prime \prime} \beta}\left(k_{2}, k_{2}^{\prime \prime} ; P_{23}\right) \\
& \times G_{\beta \beta^{\prime}}\left(P_{23}-k_{2}^{\prime \prime}\right) M_{\lambda_{2}^{\prime \prime} \beta^{\prime}, \lambda_{2}^{\prime} \alpha^{\prime}}\left(k_{2}^{\prime \prime}, k_{2}^{\prime} ; P_{23}\right) .
\end{aligned}
$$

Note that the total pair momentum $P_{23}=k_{2}+k_{3}=P-k_{1}$ depends now on the spectator momentum $k_{1}$, which implies that the $N N$ amplitude $M_{\lambda_{2} \alpha, \lambda_{2}^{\prime} \alpha^{\prime}}\left(k_{2}, k_{2}^{\prime} ; P_{23}\right)$ has to be known in arbitrary reference frames. However, because of the CST equation's manifest covariance it is sufficient to calculate the two-body amplitude in its rest frame, and determine it in moving frames through the corresponding boost operations.

If $\Lambda$ is a space-time Lorentz transformation, and $S(\Lambda)$ its representation in a one-particle Dirac space, satisfying

$S^{-1}(\Lambda) \gamma^{\mu} S(\Lambda)=\Lambda^{\mu}{ }_{v} \gamma^{\nu}$

then $M$ transforms like

$M_{\alpha \alpha^{\prime}, \beta \beta^{\prime}}\left(\Lambda k_{2}, \Lambda k_{2}^{\prime} ; \Lambda P_{23}\right)=$

$S_{\alpha \alpha_{1}}(\Lambda) S_{\beta \beta_{1}}(\Lambda) M_{\alpha_{1} \alpha_{2}, \beta_{1} \beta_{2}}\left(k_{2}, k_{2}^{\prime} ; P_{23}\right) S_{\alpha_{2} \alpha^{\prime}}^{-1}(\Lambda) S_{\beta_{2} \beta^{\prime}}^{-1}(\Lambda)$.

All relativistic effects can be calculated exactly in CST, and the full Dirac structure of the nucleons is also taken into account.

The $3 N$ equation (17) was solved numerically for the first time in Ref. [6], for a family of older OBE potentials. Since then, much progress has been made in the development of more accurate CST $N N$ interaction models, which will be described in the following section.

\subsection{High-precision $n p$ kernels}

The first covariant NN OBE kernels in CST, based on the exchange of either four or six mesons, were published in Ref. [3]. After a long process of improvements of the applied numerical techniques, the structure of the kernels, and the

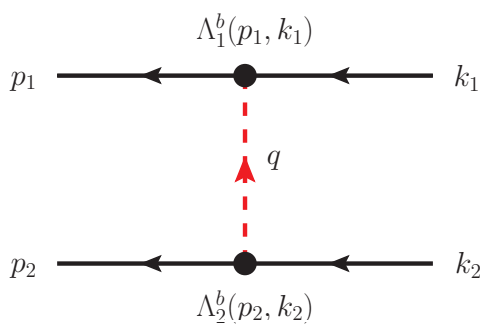

Fig. 10 The structure of a OBE kernel.

enlargement of the $n p$ data base, two new models were developed [4] whose precision in representing the most recent world $n p$ data is on par with all commonly used "realistic" potentials.

The kernels are sums of OBE contributions,

$V_{\alpha \alpha^{\prime} ; \beta \beta^{\prime}}(p, k ; P)=\sum_{b} V_{12}^{b}(p, k ; P)$.

In the notation of Fig. 10, the individual boson contributions are of the form

$V_{12}^{b}(p, k ; P)=\varepsilon_{b} \delta \frac{\Lambda_{1}^{b}\left(p_{1}, k_{1}\right) \otimes \Lambda_{2}^{b}\left(p_{2}, k_{2}\right)}{m_{b}^{2}+\left|q^{2}\right|} f\left(\Lambda_{b}, q\right)$.

Here, $b=\{s, p, v, a\}$ denotes the boson type (scalar, pseudoscalar, vector, axial vector), $q=p_{1}-k_{1}=k_{2}-p_{2}=p-k$ the momentum transfer, $m_{b}$ the boson mass, $\varepsilon_{b}$ a phase factor, and $\delta=1$ for isoscalar bosons and $\delta=\tau_{1} \cdot \tau_{2}=-1-$ $2(-)^{I}$ for isovector bosons. All boson form factors, $f$, have the simple form

$f\left(\Lambda_{b}, q\right)=\left[\frac{\Lambda_{b}^{2}}{\Lambda_{b}^{2}+\left|q^{2}\right|}\right]^{4}$,

where $\Lambda_{b}$ is the boson form factor mass. The use of the absolute value $\left|q^{2}\right|$ amounts to a covariant redefinition of the propagators and form factors in the region $q^{2}>0$. It is a significant new theoretical improvement that removes all singularities and can be justified by a detailed study of the structure of the exchange diagrams [4].

The axial vector bosons are treated as contact interactions, with a structure as in Eq. (23), but with the propagator replaced by a constant, $m_{a}^{2}+\left|q^{2}\right| \rightarrow m^{2}$, where the nucleon mass sets a convenient scale not related to a boson mass (the effective boson mass in a contact interaction is infinite). The explicit forms of the numerator functions $\Lambda_{1}^{b} \otimes \Lambda_{2}^{b}$ can be inferred from Table 1 . Note that $\lambda_{p}=0$ corresponds to pure pseudovector coupling, and that the definitions of the offshell coupling parameters $\lambda$ or $v$ differ for each boson.

The parameters of our two models, called WJC-1 and WJC-2, are displayed in Tables 2 and 3. Model WJC-1 was constructed with the goal to obtain the best possible fit, while the objective of WJC-2 was to use the smallest number of parameters without deteriorating the quality of the fit too much. 
Table 1 Mathematical forms of the $b N N$ vertex functions, with $\Theta(p) \equiv(m-\not p) / 2 m$. The vector propagator is $\Delta_{\mu v}=g_{\mu v}-q_{\mu} q_{v} / m_{v}^{2}$ with the boson momentum $q=p_{1}-k_{1}=k_{2}-p_{2}$.

\begin{tabular}{|c|c|c|c|}
\hline$J^{P}(b)$ & $\varepsilon_{b}$ & $\Lambda_{1} \otimes \Lambda_{2}$ & $\Lambda(p, k)$ or $\Lambda^{\mu}(p, k)$ \\
\hline $0^{+}(s)$ & - & $\Lambda_{1} \Lambda_{2}$ & $g_{s}-v_{s}[\Theta(p)+\Theta(k)]$ \\
\hline $0^{-}(p)$ & + & $\Lambda_{1} \Lambda_{2}$ & $\begin{array}{l}g_{p} \gamma^{5} \\
-g_{p}\left(1-\lambda_{p}\right)\left[\Theta(p) \gamma^{5}+\gamma^{5} \Theta(k)\right]\end{array}$ \\
\hline $1^{-}(v)$ & + & $\Lambda_{1}^{\mu} \Lambda_{2}^{v} \Delta_{\mu v}$ & $\begin{array}{l}g_{v}\left[\gamma^{\mu}+\frac{K_{v}}{2 M} i \sigma^{\mu v}(p-k)_{v}\right] \\
+g_{v} v_{v}\left[\Theta(p) \gamma^{\mu}+\gamma^{\mu} \Theta(k)\right]\end{array}$ \\
\hline $1^{+}(a)$ & + & $\Lambda_{1}^{\mu} \Lambda_{2}^{v} g_{\mu v}$ & $g_{a} \gamma^{5} \gamma^{v}$ \\
\hline
\end{tabular}

Table 2 Values of the 27 parameters for WJC-1 with 7 bosons and 2 axial vector contact interactions. All masses and energies are in $\mathrm{MeV}$; other couplings are dimensionless; $G_{b}=g_{b}^{2} / 4 \pi$. Parameters in bold were varied during the fit; those labeled with an ${ }^{*}$ were constrained to equal the one above. The triton binding energy is $E_{t}$ (with its experimental value in parentheses).

\begin{tabular}{lcccrcc}
\hline$b$ & $\mathrm{I}$ & $G_{b}$ & $m_{b}$ & $\lambda_{b}$ or $v_{b}$ & $\kappa_{v}$ & $\Lambda_{b}$ \\
\hline$\pi^{0}$ & 1 & $\mathbf{1 4 . 6 0 8}$ & 134.9766 & $\mathbf{0 . 1 5 3}$ & - & $\mathbf{4 4 0 0}$ \\
$\pi^{ \pm}$ & 1 & $\mathbf{1 3 . 7 0 3}$ & 139.5702 & $-\mathbf{0 . 3 1 2}$ & - & $4400^{*}$ \\
$\eta$ & 0 & $\mathbf{1 0 . 6 8 4}$ & $\mathbf{6 0 4}$ & $\mathbf{0 . 6 2 2}$ & - & $4400^{*}$ \\
$\sigma_{0}$ & 0 & $\mathbf{2 . 3 0 7}$ & $\mathbf{4 2 9}$ & $-\mathbf{1 5 . 1 6 9}$ & - & $\mathbf{1 4 3 5}$ \\
$\sigma_{1}$ & 1 & $\mathbf{0 . 5 3 9}$ & $\mathbf{5 1 5}$ & $\mathbf{4 . 7 6 3}$ & - & $1435^{*}$ \\
$\omega$ & 0 & $\mathbf{3 . 4 5 6}$ & $\mathbf{6 5 7}$ & $\mathbf{0 . 8 4 3}$ & $\mathbf{0 . 0 4 8}$ & $\mathbf{1 3 7 6}$ \\
$\rho$ & 1 & $\mathbf{0 . 3 2 7}$ & $\mathbf{7 8 7}$ & $-\mathbf{1 . 2 6 3}$ & $\mathbf{6 . 5 3 6}$ & $1376^{*}$ \\
$h_{1}$ & 0 & $\mathbf{0 . 0 0 2 6}$ & - & - & - & $1376^{*}$ \\
$a_{1}$ & 1 & $-\mathbf{0 . 4 3 6}$ & - & - & - & $1376^{*}$ \\
\hline \multicolumn{7}{c}{$\Lambda_{N}=\mathbf{1 6 5 6} ; E_{t}=-8.48(-8.48)$} \\
\hline \multicolumn{7}{c}{}
\end{tabular}

Table 3 Values of the 15 parameters for WJC-2 with 7 bosons. See the caption to Table 2 for further explanation.

\begin{tabular}{lccrrrc}
\hline$b$ & $\mathrm{I}$ & $G_{b}$ & $m_{b}$ & $\lambda_{b}$ or $v_{b}$ & $\kappa_{v}$ & $\Lambda_{b}$ \\
\hline$\pi^{0}$ & 1 & $\mathbf{1 4 . 0 3 8}$ & 134.9766 & 0.0 & - & $\mathbf{3 6 6 1}$ \\
$\pi^{ \pm}$ & 1 & $14.038^{*}$ & 139.5702 & 0.0 & - & $3661^{*}$ \\
$\eta$ & 0 & $\mathbf{4 . 3 8 6}$ & 547.51 & 0.0 & - & $3661^{*}$ \\
$\sigma_{0}$ & 0 & $\mathbf{4 . 4 8 6}$ & $\mathbf{4 7 8}$ & $-\mathbf{2 . 5 9 4}$ & - & $3661^{*}$ \\
$\sigma_{1}$ & 1 & $\mathbf{0 . 4 7 7}$ & $\mathbf{4 5 4}$ & $\mathbf{9 . 8 7 5}$ & - & $3661^{*}$ \\
$\omega$ & 0 & $\mathbf{8 . 7 1 1}$ & 782.65 & 0.0 & 0.0 & $\mathbf{1 5 9 1}^{*}$ \\
$\rho$ & 1 & $\mathbf{0 . 6 2 6}$ & 775.50 & $-\mathbf{2 . 7 8 7}$ & $\mathbf{5 . 0 9 9}$ & $1591^{*}$ \\
\hline \multicolumn{7}{c}{$\Lambda_{N}=\mathbf{1 7 3 9} ; E_{t}=-8.50(-8.48)$} \\
\hline
\end{tabular}

Table 4 Comparison of precision $n p$ models and the 1993 Nijmegen phase shift analysis. The first column specifies the model, the second the number of adjustable parameters (in the case of the first four models for both $n p$ and $p p$ data), and the third the year of the data base (data prior to this year are included). Columns four to six are the obtained $\chi^{2} / N_{\text {data }}$ for various data bases (identified by their year), where the number of included data is given in parentheses. Our calculations are in bold face.

\begin{tabular}{lcc|ccc}
\hline \multicolumn{3}{c}{ Model } & \multicolumn{3}{c}{$\chi^{2} / N_{\text {data }}\left(N_{\text {data }}\right)$} \\
\hline Reference & $N_{\text {pars }}$ & Year & 1993 & 2000 & 2007 \\
\hline PWA93[7] & 39 & 1993 & $0.99(2514)$ & - & - \\
& & & $\mathbf{1 . 0 9}(3011)$ & $\mathbf{1 . 1 2}(3336)$ & $\mathbf{1 . 1 3}(3788)$ \\
Nijm I[8] & 41 & 1993 & $1.03(2514)$ & - & - \\
AV18[9] & 40 & 1995 & $1.06(2526)$ & - & - \\
CD-Bonn[10] & 43 & 2000 & - & $1.02(3058)$ & - \\
WJC-1 & 27 & 2007 & $\mathbf{1 . 0 3 ( 3 0 1 1 )}$ & $\mathbf{1 . 0 5}(3336)$ & $\mathbf{1 . 0 6}(3788)$ \\
WJC-2 & 15 & 2007 & $\mathbf{1 . 0 9}(3011)$ & $\mathbf{1 . 1 1}(3336)$ & $\mathbf{1 . 1 2}(3788)$ \\
\hline
\end{tabular}

Table 4 shows that we achieved excellent fits for the most complete data base of $n p$ scattering, and with a considerably smaller number of adjustable parameters than other realistic potential models. In fact, in view of the $\chi^{2} / N_{\text {data }}=$ 1.06 of model WJC-1, the corresponding phase shifts can be considered a new phase shift analysis which includes many more data than the "standard" Nijmegen 93 analysis to which all realistic potential models were fitted.

We emphasize that both of our models are true OBE models, proving that OBE models not only work, but can provide an excellent description of the data. This is compatible with the large $N_{c}$ limit of QCD. Furthermore, it seems likely that the efficiency of our models (precision fits with fewer parameters) may be attributed to the efficiency of the Dirac equation, as mentioned in the Introduction.

The phase shifts of models WJC-1 and WJC-2 are shown in Fig. 11, together with those of the Nijmegen phase shift analysis. While overall the three cases lie close together, it is perhaps surprising that in some regions the agreement between our models and the Nijmegen phase shifts is not better. We can conclude that our new phase shifts provide a superior representation of the data and should be used in future comparisons of model predictions with experiment. Note that our phase shifts can be used outside the framework of CST, just like any other phase shift analysis.

\subsection{Deuteron}

The deuteron binding energy was used as a constraint during the fitting of the CST NN kernels, and therefore they reproduce the experimental binding energy of $E_{d}=2.2246 \mathrm{MeV}$ automatically. The bound-state equation is derived from the representation of the CST scattering amplitude near the deuteron pole,

$$
\begin{aligned}
& M_{\lambda_{1} \lambda_{1}^{\prime}, \beta \beta^{\prime}}\left(p, p^{\prime} ; P\right)=R_{\lambda_{1} \lambda_{1}^{\prime}, \beta \beta^{\prime}}\left(p, p^{\prime} ; P\right) \\
& -\sum_{\lambda_{d}} \frac{\mathscr{G}_{\lambda_{1} \beta}\left(p, P, \lambda_{d}\right) \overline{\mathscr{G}}_{\lambda_{1}^{\prime} \beta^{\prime}}\left(p^{\prime}, P, \lambda_{d}\right)}{M_{d}^{2}-P^{2}-i \varepsilon},
\end{aligned}
$$

where $\lambda_{1}, \lambda_{1}^{\prime}$ are the helicities of the outgoing and incoming on-shell particle $1, \beta, \beta^{\prime}$ are the Dirac indices of the offshell particle $2, \lambda_{d}$ is the helicity of the deuteron, $\mathscr{G}$ is the $d n p$ vertex function [contracted with $\bar{u}\left(\boldsymbol{p}_{1}, \lambda_{1}\right)$ ] describing the coupling of the deuteron to the neutron and proton, and $R$ is a remainder function finite at the pole. The equation for $\mathscr{G}$ is found by substituting (25) into the scattering equation (10) and demanding that it hold at the pole, giving

$$
\begin{array}{r}
\mathscr{G}_{\lambda_{1} \beta}\left(p, P, \lambda_{d}\right)=-\int \frac{d^{3} k_{1}}{(2 \pi)^{3}} \frac{m}{E_{k_{1}}} \bar{V}_{\lambda_{1} \lambda_{1}^{\prime}, \beta \alpha}(p, k ; P) \\
\times G_{\alpha \beta^{\prime}}\left(k_{2}\right) \mathscr{G}_{\lambda_{1}^{\prime} \beta^{\prime}}\left(k, P, \lambda_{d}\right) .
\end{array}
$$

These vertex functions can be related to the well-known nonrelativistic $\mathrm{S}$ - and D-state deuteron wave functions $u(p)$ 

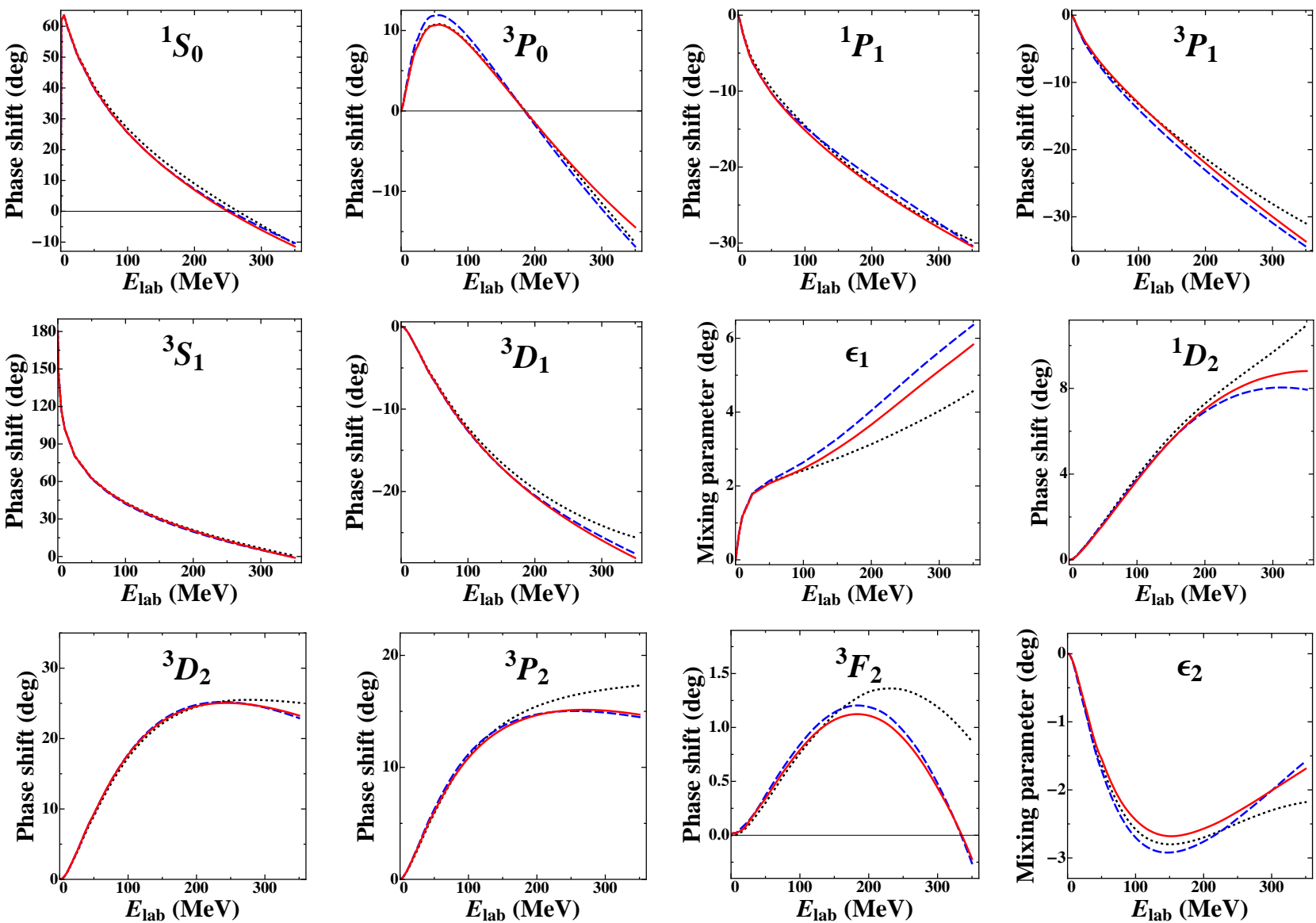

Fig. 11 Phase shifts of $n p$ scattering for partial waves with $J \leq 2$. The solid and dashed lines respresent the results of models WJC-1 and WJC-2, respectively. The dotted line shows the Nijmegen multienergy phase shift analysis of 1993[7].

Table 5 Exact and scaled deuteron probabilities (in percent) for models WJC-1 and WJC-2.

\begin{tabular}{lrrrr}
\hline & \multicolumn{2}{c}{ WJC-1 } & \multicolumn{2}{c}{ WJC-2 } \\
Probability & exact & scaled & exact & scaled \\
\hline$P_{s}$ & 97.3876 & 92.3330 & 95.7607 & 93.5985 \\
$P_{d}$ & 7.7452 & 7.3432 & 6.5301 & 6.3827 \\
$P_{v_{t}}$ & 0.1180 & 0.1119 & 0.0103 & 0.0101 \\
$P_{v_{s}}$ & 0.2234 & 0.2118 & 0.0090 & 0.0088 \\
\hline$\sum P$ & 105.4743 & 100.0000 & 102.3101 & 100.0000 \\
$\left\langle V^{\prime}\right\rangle$ & -5.4743 & - & -2.3101 & - \\
\hline total & 100.0000 & - & 100.0000 & - \\
\hline
\end{tabular}

and $w(p)$, respectively. In addition one obtains spin singlet and triplet $\mathrm{P}$-waves, $v_{s}(p)$ and $v_{t}(p)$, which are of relativistic origin. They satisfy the normalization condition

$\int_{0}^{\infty} p^{2} d p\left[u^{2}(p)+w^{2}(p)+v_{s}^{2}(p)+v_{t}^{2}(p)\right]+\left\langle V^{\prime}\right\rangle=1$

where $\left\langle V^{\prime}\right\rangle$ is a wave function average of the derivative of the kernel with respect to the total energy, evaluated at the pole energy $M_{d}$.

Table 5 shows the individual contributions to the normalization of Eq. (27) for the two models WJC-1 and WJC-2.
In both cases, the derivative of the kernel yields a negative result, such that the sum of the wave function probabilities by themselves exceeds 1 .

It is probably not completely consistent to use these wave functions outside CST. Nevertheless, if approximate results are deemed sufficient, the best way to do so appears to be to neglect the derivative term and to rescale the wave functions to a total probability of 1 , as indicated in the Tab. 5 .

Tables with the numerical values, and convenient parameterizations using analytic functions, both in momentum and coordinate space, are given in Ref. [11].

3.5 Three-nucleon binding energy and three-nucleon forces

One of the few persistent problems of low-energy few-nucleon physics is the apparent inability of realistic $N N$ potentials to explain the experimental triton binding energy $E_{t}=8.48 \mathrm{MeV}$. The potentials with the best fit to the $N N$ data yield binding energies roughly between 7.6 and $8 \mathrm{MeV}$.

The view commonly adopted in order to deal with this discrepancy is that $3 N$ forces must be an essential part of $3 N$ dynamics and must be included. Models for $3 N$ forces 

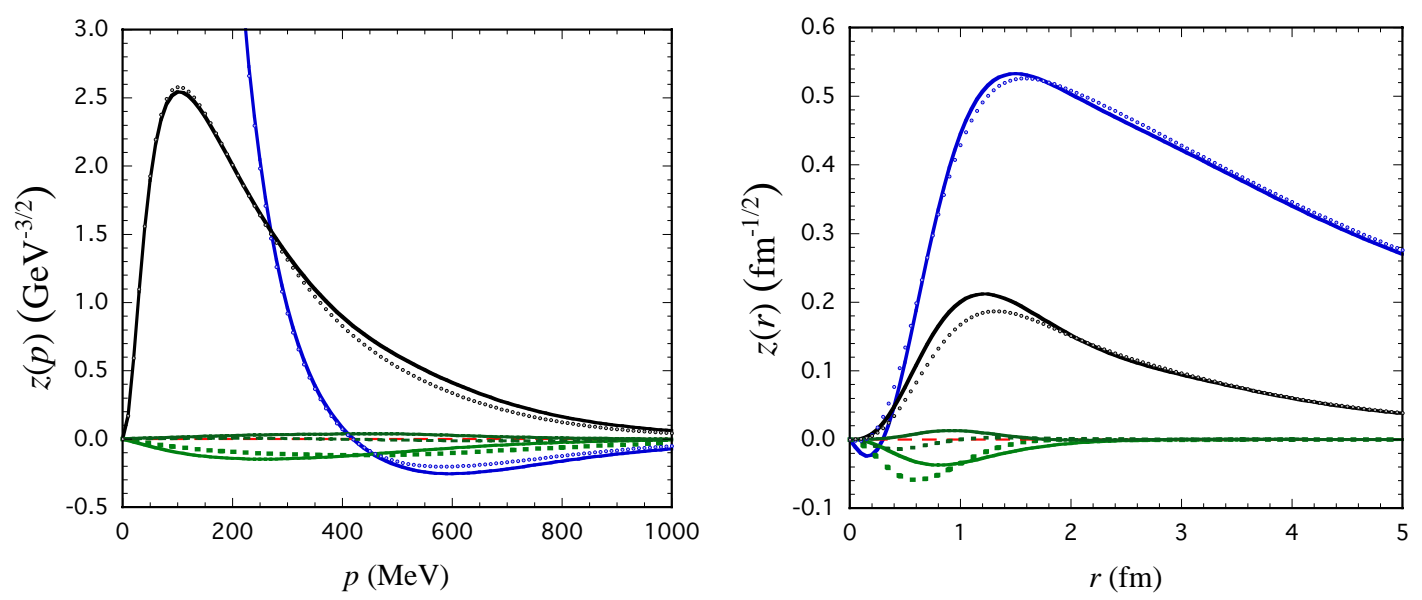

Fig. 12 Deuteron wave functions (generically denoted as $z$ ) in momentum space (left panel) and in coordinate space (right panel). The largest two wave functions are $u$ and $w$ (with $u$ reaching the highest values), with solid lines for WJC-1 and dotted lines for WJC-2. The small P-waves $v_{t}$ are solid for both models, WJC-1 being negative and WJC-2 positive, whereas both dotted lines are for $v_{s}$, with WJC-1 negative and WJC-2 closer to zero.

introduce additional parameters, which are usually adjusted to reproduce the triton binding energy. Of course, in doing so one gives up on the challenge to actually predict it. Moreover, it raises serious problems of consistency between $2 \mathrm{~N}$ and $3 N$ force models. For instance, when the meson-nucleon cut-off parameters employed in realistic potentials are used for the same vertices in $3 N$ forces, they lead to very strong overbinding.

Relativity was considered another possible candidate to resolve-at least in part- the triton binding-energy problem. However, the first results with CST OBE potentials were not encouraging, with model IIA of Ref. [3] yielding only $E_{t} \sim 6 \mathrm{MeV}$.

The older $N N$ kernels of Ref. [3] did not yet exploit the full complexity of boson-nucleon vertices possible in a theory with off-mass-shell nucleons. First steps to explore these additional types of coupling were taken by including terms proportional to $v_{s}$ in the vertices for the coupling of scalar mesons to nucleons [6], in the form

$g_{s} \Lambda(p, k)=g_{s} \mathbb{1}-\frac{v_{s}}{2 m}[(m-\not p)+(m-\not k)]$.

They contribute only when the incoming or outgoing nucleon is off mass shell, since $(m-\not p) u(\mathbf{p}, \lambda)=0$ for a nucleon with on-shell momentum $p$.

In the models of Ref. [6], the off-shell coupling constants $v_{\sigma}$ and $v_{\delta}$ of the two included scalar bosons-an isoscalar $\sigma$ and an isovector $\delta$ meson-were not allowed to vary independently. During the initial exploratory fits their ratio had emerged as approximately constant, and to keep the number of independent parameters small we chose to express the individual coupling constants as multiples of a single parameter $v$, in the form $v_{\sigma}=-0.75 g_{\sigma} v$ and $v_{\delta}=$ $2.6 g_{\delta} v$.

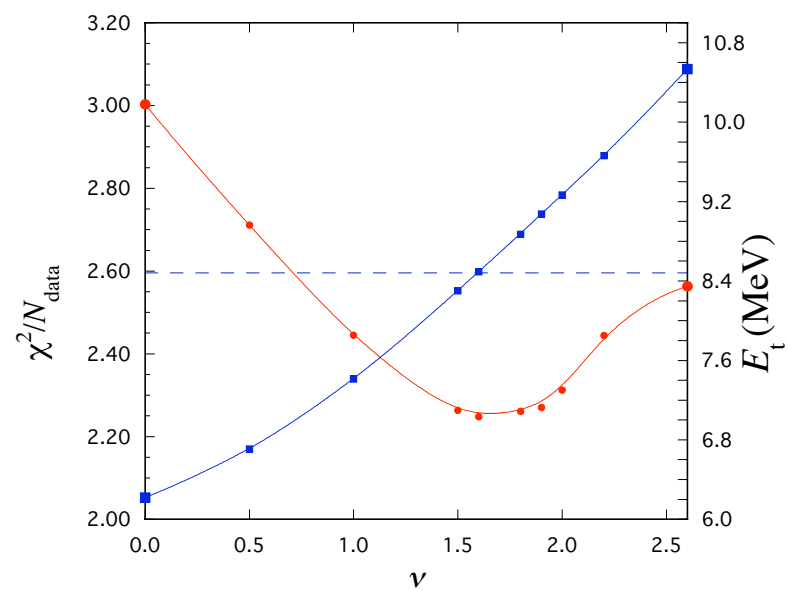

Fig. $13 \chi^{2} / N_{\text {data }}$ for a $1994 n p$ data base and triton binding energy for a family of $N N$ models (Wxx) with scalar off-shell coupling, characterized by the strength parameter $v$. Both curves are smooth fits through the points.

It turned out that the scalar off-shell coupling term in Eq. (28) has very important effects, both in the two- and threenucleon sector. It improves the fit to the $N N$ data considerably, and contributes strongly to the $3 N$ binding energy. The best fit to the data was obtained with $v=1.6$, and the corresponding $N N$ potential received the name W16. Remarkably, the triton binding energy for this model is $E_{t}=8.489$ $\mathrm{MeV}$, which coincides almost exactly with the experimental value.

To investigate the importance of the scalar off-shell coupling in more detail, we constructed a family of $N N$ kernels in which the parameter $v$ was fixed at specific values while the other potential parameters were varied to give the best fit. Figure 13 and-for a few selected models-Table 6 demonstrate that the $\chi^{2} / N_{\text {data }}$ clearly deteriorates when $v$ is 

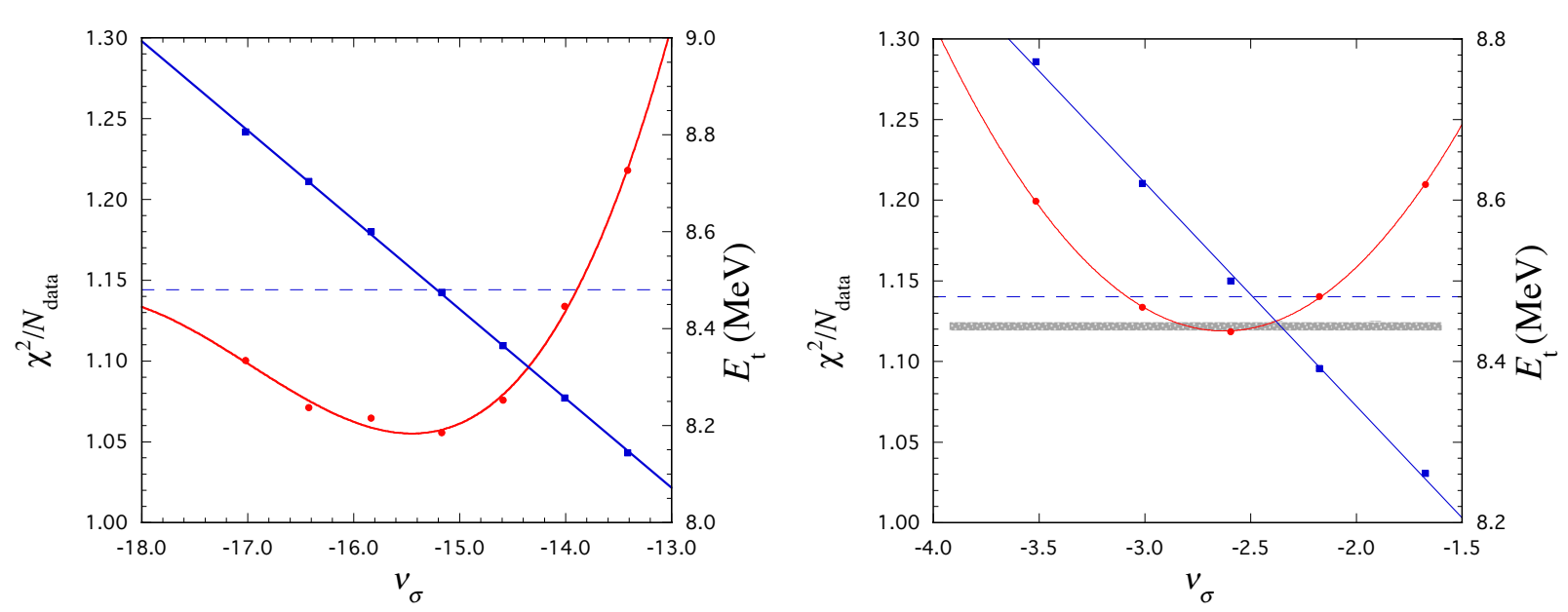

Fig. 14 Results for calculations of $\chi^{2} / N_{\text {data }}$ (red points and left scale) to a $2007 n p$ data base, and triton binding energy $E_{t}$ (blue points and right scale) for WJC-1 family (left panel) and for WJC-2 family of models (right panel). The points with the lowest $\chi^{2} / N_{\text {data }}$ are models WJC-1 and WJC-2, respectively. The other models of the two families were obtained by fixing $v_{\sigma}$ at different values and refitting all other parameters. The curves are fits through the actually calculated points. These fits are linear for $E_{t}$ in both cases, while the fit to $\chi^{2} / N_{\text {data }}$ is cubic for WJC-1 and quadratic for WJC-2.

Table 6 Scalar meson parameters of the $N N$ potential models used in the calculations of the $3 N$ vertex functions. Also given are the $\chi^{2} / \mathrm{N}_{\text {data }}$ of the models obtained in fits to a $N N$ data base of 1994, and the corresponding triton binding energies $E_{t}$. All masses and binding energies are in $\mathrm{MeV}$.

\begin{tabular}{cccccc}
\hline & W00 & W10 & W16 & W19 & W26 \\
\hline$v$ & 0.0 & 1.0 & 1.6 & 1.9 & 2.6 \\
$g_{\sigma}^{2} / 4 \pi$ & 5.84067 & 5.50753 & 4.99887 & 4.67948 & 4.05718 \\
$v_{\sigma}$ & 0.0 & -0.75 & -1.2 & -1.425 & -1.95 \\
$m_{\sigma}$ & 525 & 515 & 506 & 501 & 491 \\
$g_{\delta}^{2} / 4 \pi$ & 0.14812 & 0.69046 & 0.62818 & 0.47598 & 0.25045 \\
$v_{\delta}$ & 0.0 & 2.6 & 4.16 & 4.94 & 6.76 \\
$m_{\delta}$ & 390 & 540 & 512 & 474 & 399 \\
\hline$\chi^{2} / \mathrm{N}_{\text {data }}$ & 3.00 & 2.45 & 2.25 & 2.27 & 2.56 \\
$E_{t}$ & 6.217 & 7.411 & 8.489 & 9.072 & 10.533 \\
\hline & & & & &
\end{tabular}

moved away from its preferred value, while at the same time $E_{t}$ changes rapidly.

These results established that off-shell coupling of scalar bosons to nucleons is a very important mechanism of an OBE $N N$ interaction, which strongly influences the $3 N$ binding energy, although the fact that the experimental value of $E_{t}$ is reproduced without $3 N$ forces might be considered just an interesting coincidence.

After the fits of the new high-precision models WJC1 and WJC-2 were completed, it came as a surprise that both models-with $E_{t}=8.48 \mathrm{MeV}$ and $E_{t}=8.50 \mathrm{MeV}$, respectively - again predict the experimental binding energy very closely, even though their detailed structure and their parameters differ quite significantly from each other and from the old model W16. The result is robust, and it becomes difficult to believe in a mere coincidence! Figure 14 shows again the changes in $\chi^{2} / N_{\text {data }}$ and $E_{t}$ when $v_{\sigma}$ is held fixed at certain values while all other potential parameters (this time including $v_{\delta}$ ) are refitted.

One may wonder whether this is really such a satisfying result, because surely $3 N$ forces still need to be included, which will most likely spoil the nice agreement with the experimental value for $E_{t}$.

The answer, surprising perhaps, is no: in a true relativistic OBE theory for the interaction between nucleons no additional irreducible $3 \mathrm{~N}$ forces can be derived from the basic vertices of the theory!

To see this, consider the diagrams of Fig. 15: diagram (a) is reducible, but the "active" nucleon in the middle is off mass shell and can be in a negative-energy state. The corresponding Z-graphs contribute to a $3 N$ force in nonrelativistic frameworks, but in CST it is automatically included at OBE level. The lowest-order irreducible $3 N$ force contributions in CST are shown in diagrams (b) and (c) of Fig. 15. However, diagrams (a) and (b) of Fig. 16 are of the same order and would have to be included as well for consistency. The latter are reducible $3 \mathrm{~N}$ forces, generated by iteration once next-tolowest order diagrams (b) and (c) of Fig. 17 are included in the $N N$ kernel. But they are not present at the order of OBE, which is defined by Fig. 17(a) alone. Consequently, neither Fig. 15(b) nor (c) is present in an OBE theory.

There is a possibility that other degrees of freedom may play a role, such as intermediate-state excitations of the nucleon. Figure 18 (a) shows the $2 \pi$-exchange $3 N$ force generated by the virtual excitation of a nucleon to a $\Delta$ isobar, the well-known Fujita-Miyazawa force [12]. Such an explicit inclusion of the $\Delta$ or other nucleon resonances goes beyond the scope of our current $N N$ theory.

However, there is some evidence that this kind of $3 N$ forces would not alter the picture very much: 


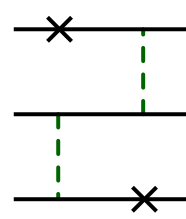

(a)

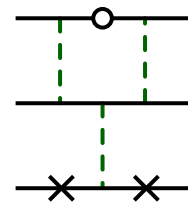

(b)

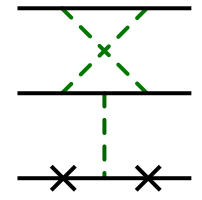

(c)
Fig. 15 Examples of $3 N$ forces in the CST. Diagram (a) is reducible, and generated automatically through the iteration of the OBE kernel. Diagrams (b) and (c) are irreducible relativistic $3 N$ forces, but contribute only in higher-than OBE order. An " $\mathrm{x}$ " on a line indicates the particle is on its positive-energy mass shell. An open circle is the remainder of the diagram once the on-mass-shell contribution has been subtracted.

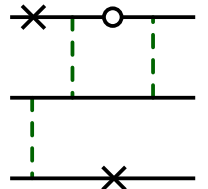

(a)

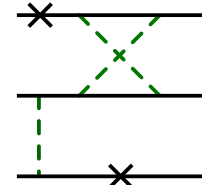

(b)
Fig. 16 Examples of reducible $3 N$ forces at next-to-lowest order of the $N N$ kernel in the CST.

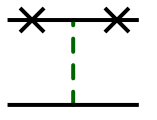

(a)

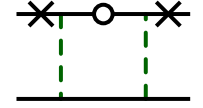

(b)

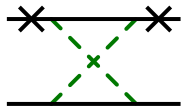

(c)
Fig. 17 Diagrams of the CST $N N$ kernel up to 2nd order. Diagram (a) is the OBE approximation, diagrams (b) and (c) are next-to-leading order corrections, not included in an OBE theory.

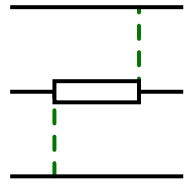

(a)

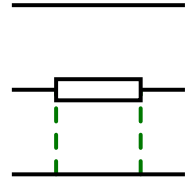

(b)
Fig. 18 Fujita-Miyazawa $3 N$ force (a), and dispersive contribution (b) of the $\Delta$ to the $N N$ interaction in the presence of a spectator nucleon.

(i) In their pioneering work on the effects of explicit $\Delta$ isobars in the $N N$ - and $3 N$-systems, Hajduk and Sauer [13, 14] found that a large part of the Fujita-Miyazawa force of Fig. 18(a) is canceled by the so-called "dispersive" contribution of the $\Delta$ to the $N N$ interaction in the presence of a spectator nucleon, as shown in Fig. 18(b), leaving only a small remainder. Subsequent work by Picklesimer et al. [15] found an almost vanishing overall effect once multi- $\Delta$ configurations are also taken into account.

(ii) Peña, Gross, and Surya [16] investigated the $2 \pi$ exchange potential of Fig. 19(a), where a subtracted covariant $\pi N$ scattering amplitude is inserted into a box diagram, containing $\Delta$, Roper, and $D_{13}$ isobars, contact terms, and offshell (non-pole) dressed nucleon terms, while the spectator nucleon is on mass shell. Embedded in a space of three nu-

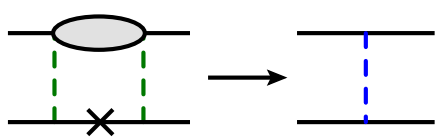

(a)

(b)

Fig. 19 The $2 \pi$-exchange potential based on a $\pi N$ scattering amplitude (shaded blob) inserted into a box diagram (a) can be very well approximated by an effective OBE kernel with $\sigma$ and $\delta$ exchange (b)

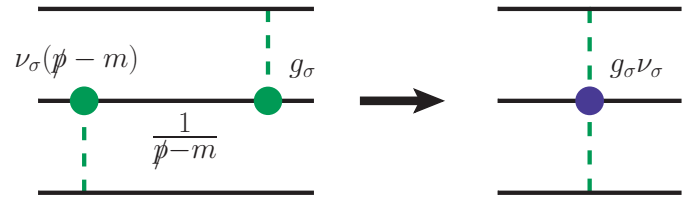

Fig. 20 Boson-nucleon vertices with off-shell coupling can generate effective $3 N$ forces. In this example, an off-shell nucleon consecutively exchanges a scalar $\sigma$ meson with two different nucleons. When a scalar off-shell vertex is multiplied with the nucleon propagator, the two separate boson-nucleon vertices shrink to a single contact vertex, and the whole diagram takes on the form of a $3 N$ force.

cleons, $3 N$ forces are generated by this $\pi N$ scattering amplitude which include - but are more general than - the FujitaMiyazawa force. They found that this $2 \pi$-exchange $N N$ potential can be very well approximated by the exchange of effective single $\sigma$ and $\delta$ mesons. In other words, a simple OBE kernel is able to represent most of the $2 \pi$-exchange dynamics, and one might expect the same to be true for the corresponding $3 N$ force.

When discussing $3 \mathrm{~N}$ forces it is important to keep in mind that it is a framework-dependent concept. We have argued that no irreducible $3 N$ forces are included in our $3 N$ calculations. However, they contain many processes that in a nonrelativistic theory could only be realized as $3 N$ forces. One example was the negative-energy part of the intermediate off-shell nucleon in Fig. 15(a). But a whole new kind of $3 N$ forces in this sense are also generated by boson-nucleon vertices with off-shell coupling. As illustrated in Fig. 20, such vertices with off-shell terms together with off-shell propagators can transform into contact vertices and take the form of $3 N$ forces. If the two bosons emerging from the point vertex both couple to the same instead of to different nucleons, they also contribute to a new two-boson-exchange $N N$ force. In fact, this process can happen arbitrarily often, such that multi-boson exchange two- and three-body forces are produced.

Note that these " $3 N$ forces," which are reducible in the framework of the CST and automatically included in a OBE model, contain no new parameters and are completely determined from the $N N$ interaction. Our calculations provide true predictions of the triton binding energy and of the structure of the $3 N$ bound state in the form of the $3 N$ vertex function. In order to test these vertex functions, we turn now our attention to the electromagnetic form factors of the $3 \mathrm{~N}$ bound states. 

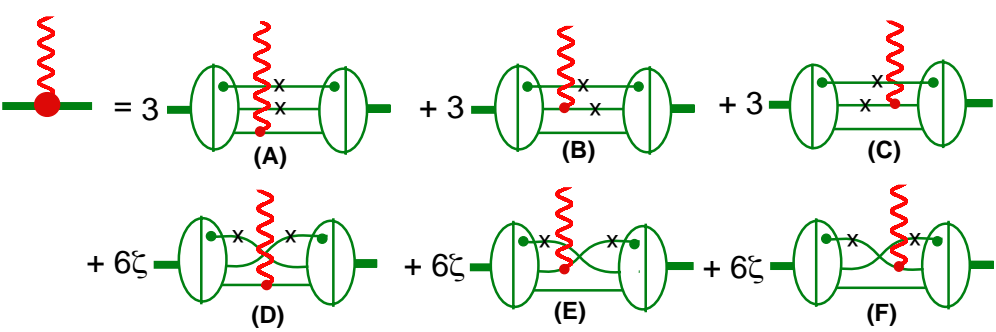

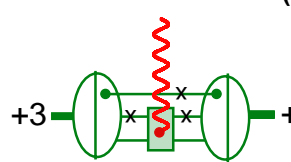

(G)

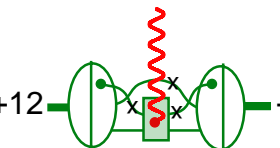

(H)

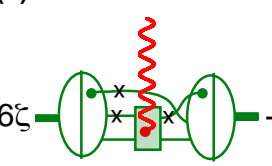

(I)

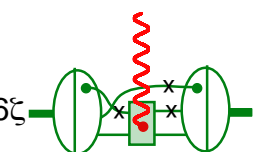

(J)
Fig. 21 The electromagnetic $3 N$ current in CST for elastic electron scattering from the $3 N$ bound state. A cross on a nucleon line indicates that the particle is on mass shell. Diagrams (A) to (F) define the complete impulse approximation (CIA), in which the photon couples to single nucleons, which can be off-shell (A and D) or on-shell before or after the photon-nucleon vertex $(\mathrm{B}, \mathrm{C}, \mathrm{E}$, and F). The approximation denoted CIA-0 replaces the vertex function with two nucleons off mass shell in diagrams $(B),(C),(E)$, and $(F)$ by a vertex function with only one nucleon off mass shell. The interaction diagrams $(\mathrm{G})$ to $(\mathrm{J})$ describe processes in which the photon couples to two-body currents associated with the two-nucleon kernel.

\section{Electromagnetic three-nucleon form factors}

The electromagnetic form factors of nuclei provide important information about their internal structure. They have been used extensively in order to test models of the nuclear dynamics and of the associated electromagnetic currents. While the interaction of nuclei with electromagnetic probes introduces some new problems in the form of ambiguities in the definition of the nuclear currents, the calculation of the electromagnetic $3 N$ form factors certainly presents an interesting and necessary test of the three-body CST.

\subsection{Family Wxx of $v$-dependent models in CIA}

In Ref. [17], Gross and Riska gave a general prescription for how to construct a conserved two-body current, even when the phenomenological boson form factors $f\left(\Lambda_{b}, q\right)$ attached to individual OBE diagrams are all different. To carry out this development for the dressed nucleon propagators (12), the electromagnetic one-nucleon current must be modified. When used with a conserved electron current, it can be written

$$
\begin{aligned}
j_{N}^{\mu}\left(k^{\prime}, k\right) & =f_{0}\left(k^{\prime 2}, k^{2}\right) F_{1 N}\left(Q^{2}\right) \gamma^{\mu} \\
& +f_{0}\left(k^{\prime 2}, k^{2}\right) F_{2 N}\left(Q^{2}\right) \frac{i \sigma^{\mu v} q_{v}}{2 m} \\
& +g_{0}\left(k^{\prime 2}, k^{2}\right) F_{3 N}\left(Q^{2}\right) \Lambda_{-}\left(k^{\prime}\right) \gamma^{\mu} \Lambda_{-}(k),
\end{aligned}
$$

where we adopt the usual convention $Q^{2}=-q^{2}$ for the momentum transfer squared. The nucleon off-shell form factors $f_{0}$ and $g_{0}$ are known functions of the nucleon form factor $h$, and $F_{1 N}$ and $F_{2 N}$ are the usual electromagnetic Dirac and Pauli form factors. Since $\Lambda_{-}(k)=(m-k) / 2 m$ projects onto negative energy states, the form factor $F_{3 N}$ belongs to a term that contributes only if the nucleon is off-shell both before and after the photon-nucleon vertex.

The extension of this work to the three body sector is described in Refs. [18-20]. The form that was found for the exact CST $3 N$ electromagnetic current is shown diagramatically in Fig. 21. The first 6 terms [diagrams (A) - (F)] are referred to as the "Complete Impulse Approximation" (CIA). Note that both the complete current and CIA by itself conserve current.

The $3 N$ form factors were calculated in the CIA approximation for the first time in Ref. [21]. This calculation explored the model-dependence of the CST predictions using the family of $v$-dependent $N N$ models (here generically called "Wxx") of Tab. 6. It also compared the predictions of the best model W16 with the calculations of the PisaJlab collaboration, described in Refs. [22,23] and labeled "IARC" below. The IARC calculations use a nonrelativistic impulse approximation with a one-nucleon current and wave functions obtained from the Argonne AV18 NN and Urbana IX $3 N$ potentials, and also include first-order relativistic corrections. Since the CST calculations do not include the Coulomb force in the $p p$ interaction, L. Marcucci provided IARC calculations in which the $p p$ Coulomb interaction was turned off [24], making it possible to compare the CIA and IARC calculations more reliably (since both now give ${ }^{3} \mathrm{H}$ and ${ }^{3} \mathrm{He}$ wave functions with the same binding energies). Some of the results for the isoscalar and isovector $3 N$ charge and magnetic form factors are shown in Fig. 22.

This figure shows, first of all, that the overall behavior of the CST results is very reasonable. No pathological structures are introduced by the scalar off-shell interactions, and the CST description of the $3 N$ bound state appears to be perfectly valid.

Almost nothing was known about the off-shell form factor $F_{3 N}$ (except that $F_{3 N}(0)=1$ ), but by varying its chosen functional form it was found that the $3 \mathrm{~N}$ form factors depend only very weakly on it. The small selection of results shown in Fig. 22 therefore includes only the usual on-shell current.

In CIA (where interaction currents are not included), one cannot expect a quantitative description of the experimental $3 N$ form factors. Furthermore, the term "impulse 

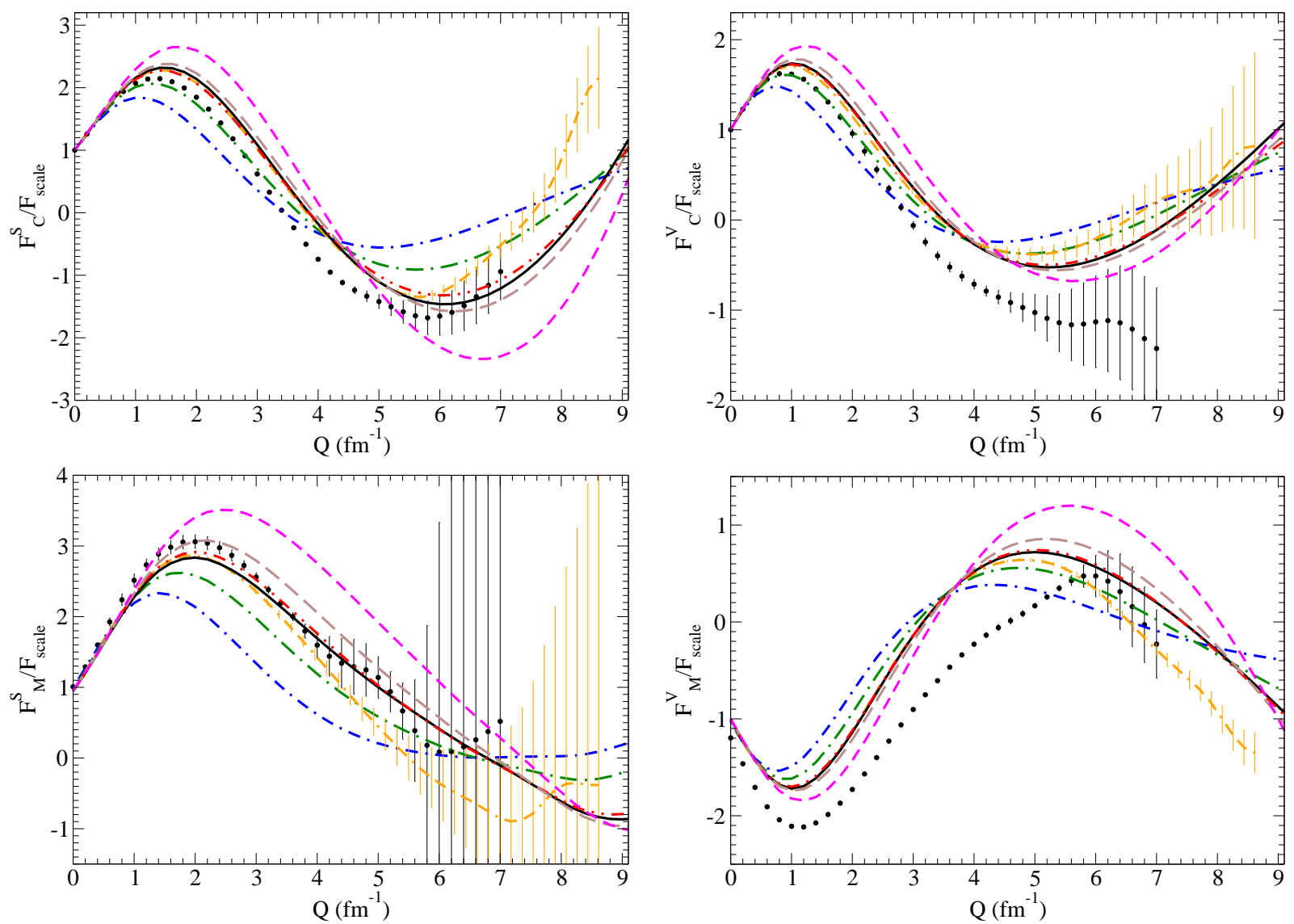

Fig. 22 Isoscalar and isovector charge (upper panel) and magnetic (lower panel) form factors of the $3 N$ bound states for different $N N$ interaction models in CIA. All form factors are divided by a simple exponential scale function defined in Ref. [21]. Shown are the results for models W00 (short dash-dotted), W10 (long dash-dotted), W16 (dash-double dotted), W19 (long dashed), and W26 (short dashed), all with the MMD nucleon form factor [25]. In addition, W16 (solid line) is compared to IARC by Marcucci (double dash-dotted), where both calculations used the Galster nucleon form factor [26]. The theoretical error bars of the Greens function Monte Carlo IARC calculations are also given. All panels also show the experimental data (full circles)[27].

approximation" can be misleading because it depends on the framework used. The CIA in CST includes contributions that in nonrelativistic frameworks appear as interaction currents (pair terms related to Z-graphs), so care has to be taken when results of different formalisms are compared.

The $3 N$ form factors obtained with model W16, which reproduces the experimental triton binding energy and comes closest to the one predicted by the nonrelativistic AV18/UIX potentials, are remarkably similar to the IARC form factors up to moderate values of the momentum transfer (around 4 to $6 \mathrm{fm}^{-1}$ ). This indicates that the $3 N$ binding energy determines their behavior to larger momentum transfer than previously expected.

It may be surprising at first that the CST W16 and IARC form factors are so close to each other, because the CIA calculations include Z-graph-type interaction currents left out from IARC. The explanation is that the Wxx family of $N N$ models uses pure pseudovector $\pi N N$ coupling, which suppresses Z-graphs.
There is one case in which a comparison of CIA results with experimental data is meaningful. The leading interaction current, the pion pair term, is almost completely absent from the isoscalar magnetic form factor. We can see in the lower left panel of Fig. 22 that W16 indeed comes relatively close to the data. The small discrepancy at low $Q$ is due to the incorrect binding energy of the ${ }^{3} \mathrm{He}$ nucleus, which, without the Coulomb interaction, is the same as for ${ }^{3} \mathrm{H}$.

\subsection{Models WJC-1 and WJC-2 in CIA-0}

After the high-precision $N N$ models WJC-1 and WJC-2 became available, the corresponding $3 N$ form factors were again calculated in CIA [28]. This time, however, for practical reasons it was necessary to apply an approximation in which the $3 N$ vertex function with two off-shell nucleons is replaced by a vertex function with only one nucleon off mass shell. This approximation, called CIA-0, can be tested with model $\mathrm{W} 16$, for which the exact result is known. 

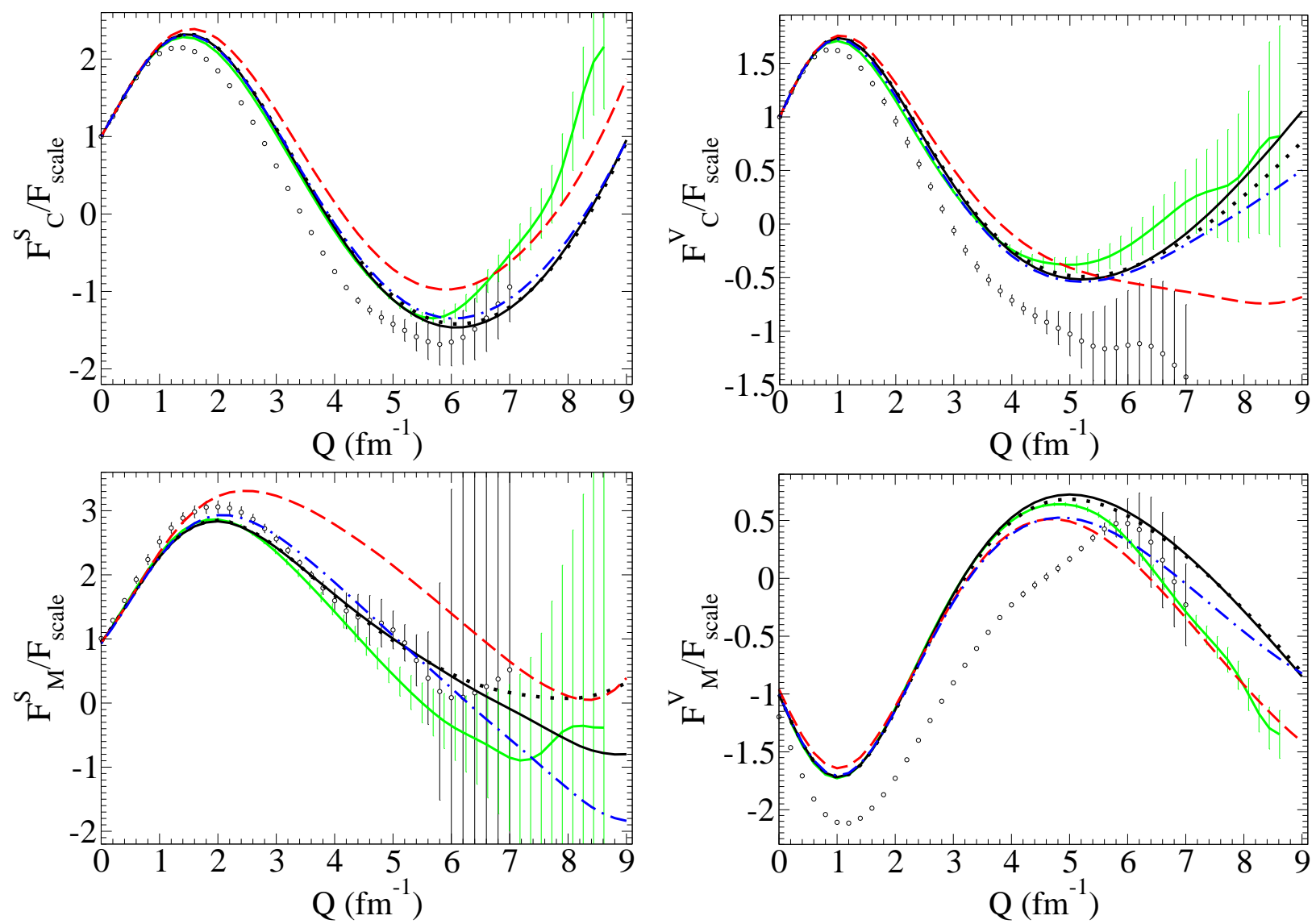

Fig. 23 Isoscalar (first row) and isovector (second row) charge form factors of the $3 N$ bound states. In each case, the form factor is divided by a common scaling function [28]. The solid line is the result for $N N$ model W16 in CIA, the dotted line is the approximation CIA-0 for the same model. The dashed line is model WJC-1, and the dash-dotted line is model WJC-2, both in CIA-0. For comparison, the solid line with theoretical error bars is the result of an IARC calculation by Marcucci [24] based on the AV18/UIX potential. All calculations employ the on-shell single-nucleon current, with the Galster parameterization of the nucleon form factors [26]. The full circles represent the experimental data [27].

Figure 23 shows again the isoscalar and isovector charge and magnetic $3 \mathrm{~N}$ form factors, this time for models WJC1 and WJC- 2 in CIA- 0 , W16 both in CIA and CIA- 0 , and IARC for the AV18/UIX interaction. We can see that CIA0 is an excellent approximation for W16, which hopefully holds also for the new models.

All models reproduce the correct $3 N$ binding energy, and the form factors remain close to each other. The only exception is WJC-1, for which some deviations are observed already at relatively small $Q$. The reason for this behavior is instructive: WJC-1 is the only model with a mixed pseudoscalar-pseudovector $\pi N N$ coupling. Its pseudoscalar part induces strong Z-graph-type currents, which are not present in the other cases.

To summarize, the $3 \mathrm{~N}$ electromagnetic form factors obtained so far in CST present a very coherent picture, from which one can conclude that CST provides a sound description of the structure of the $3 \mathrm{~N}$ bound states. For more detailed studies the interaction curents have to be calculated as well.

\section{Brief overview of other applications}

In this limited space, only a few applications of the CST could be discussed in some detail. In this section we provide a very brief (and incomplete) overview of other applications of the CST that were carried out in the past or are still under active development.

1. Electron-deuteron scattering: Deuteron form factors [29] were calculated using the earlier models developed in Ref. [3], and a number of calculations of deuteron electro-disintegration have been completed [30,31]. Calculations of the deuteron form factors using the $v$ dependent models are currently underway. It is important to see if the interaction currents predicted by the $v$ dependence of the scalar exchange couplings will have a significant effect on the observables.

2. $\pi^{0}$ production in $N N$ scattering: Nonperturbative treatment of negative energy intermediate states gave a smaller result than the perturbative calculations commonly used [32]. 
3. Pion-nucleon elastic scattering and pion photoproduction: Consistent unitary, current conserving, resonance model of $\pi N$ scattering [33] and $\pi$ photoproduction [34] including $\Delta$, Roper, and $D_{13}$ resonance contributions.

4. Structure of the nucleon, $\Delta$, and Roper electromagnetic form factors: CST calculations using fermion wave functions with a quark-diquark structure and calculating the nucleon form factors [35,36], $N \rightarrow \Delta$ transition [37, 38], $N \rightarrow$ Roper transition [39], and the $\Delta$ form factors [40,41].

5. Quark confinement and the meson spectrum: A CST model of quark-antiquark interactions, including confinement and an NJL mechanism that explains the small pion mass and allows for a scalar confinement mechanism, was applied to a description of the pion and the lightest states in the meson spectrum $[42,43]$. This model will be revised and extended to the full meson spectrum.

6. Proton-nucleus scattering: The CST was used to develop a covariant multiple scattering series $[44,45]$ which served as a justification for using the CST as a basis for a covariant description of proton scattering from nuclei $[46,47]$.

7. EMC effect: The CST can serve as a basis for a discussion and explanation of the EMC effect $[48,49]$.

Acknowledgements F. G. was supported by Jefferson Science Associates, LLC under U.S. DOE Contract No. DE-AC05-06OR23177. A. S. was supported by Fundação para a Ciência e a Tecnologia (FCT) under grant No. POCTI/ISFL/2/275

\section{References}

1. F. Gross. Phys. Rev. 186, 1448 (1969); Phys. Rev. D 10, 223 (1974); Phys. Rev. C 26, 2203 (1982)

2. F. Gross, Phys. Rev. C 26, 2226 (1982)

3. F. Gross, J.W. Van Orden, K. Holinde, Phys. Rev. C 45, 2094 (1992)

4. F. Gross, A. Stadler, Phys. Rev. C 78, 014005 (2008)

5. A. Stadler, F. Gross, M. Frank, Phys. Rev. C 56, 2396 (1997)

6. A. Stadler, F. Gross, Phys. Rev. Lett. 78, 26 (1997)

7. V.G.J. Stoks, R.A.M. Klomp, M.C.M. Rentmeester, J.J. de Swart, Phys. Rev. C 48, 792 (1993)

8. V.G.J. Stoks, R.A.M. Klomp, C.P.F. Terheggen, J.J. de Swart, Phys. Rev. C 49, 2950 (1994)

9. R.B. Wiringa, V.G.J. Stoks, R. Schiavilla, Phys. Rev. C 51, 38 (1995)

10. R. Machleidt, Phys. Rev. C 63, 024001 (2001)

11. F. Gross, A. Stadler, arXiv:1007.0778 [nucl-th]

12. J. Fujita, H. Miyazawa, Prog. Theor. Phys. 17, 360 (1957)

13. C. Hajduk, P.U. Sauer, Nucl. Phys. A 322, 329 (1979)

14. C. Hajduk, P.U. Sauer, W. Strueve, Nucl. Phys. A 405, 581 (1983)

15. A. Picklesimer, R.A. Rice, R. Brandenburg, Phys. Rev. C 45, 547 (1992)

16. M.T. Peña, F. Gross, Y. Surya, Phys. Rev. C 54, 2235 (1996)

17. F. Gross, D.O. Riska, Phys. Rev. C 36(5), 1928 (1987)

18. F. Gross, A. Stadler, M.T. Peña, Phys. Rev. C 69, 034007 (2004)

19. A.N. Kvinikhidze, B. Blankleider, Phys. Rev. C 56, 2973 (1997)
20. J. Adam, Jr., J.W. Van Orden, Phys. Rev. C 71, 034003 (2005)

21. S.A. Pinto, A. Stadler, F. Gross, Phys. Rev. C 79, 054006 (2009)

22. L.E. Marcucci, D.O. Riska, R. Schiavilla, Phys. Rev. C 58, 3069 (1998)

23. L.E. Marcucci, M. Viviani, R. Schiavilla, A. Kievsky, S. Rosati, Phys. Rev. C 72, 014001 (2005)

24. L.E. Marcucci, private communication

25. P. Mergell, U.G. Meißner, D. Drechsel, Nucl. Phys. A 596, 367 (1996)

26. S. Galster, H. Klein, J. Moritz, K.H. Schmidt, D. Wegener, J. Bleckwenn, Nucl. Phys. B 32, 221 (1971)

27. I. Sick, Prog. Part. Nucl. Phys. 47, 245 (2001)

28. S.A. Pinto, A. Stadler, F. Gross, Phys. Rev. C 81, 014007 (2010)

29. N.D. J. W. Van Orden, F. Gross, Phys. Rev. Lett. 75, 4369 (1995)

30. J. Adam, Jr., F. Gross, S. Jeschonnek, P. Ulmer, J.W.V. Orden, Phys. Rev. C 66, 044003 (2002)

31. S. Jeschonnek, J. Van Orden, Phys. Rev. C 78, 014007 (2008); C 80, 054001 (2009); C 81, 014008 (2010)

32. J. Adam Jr., A. Stadler, M.T. Peña, F. Gross, Phys. Lett. B 407, 97 (1997)

33. F. Gross, Y. Surya, Phys. Rev. C 47, 703 (1993)

34. Y. Surya, F. Gross, Phys. Rev. C 53, 2422 (1996)

35. F. Gross, P. Agbakpe, Phys. Rev. C 73, 015203 (2006)

36. F. Gross, G. Ramalho, M.T. Peña, Phys. Rev. C 77, 015202 (2008)

37. G. Ramalho, M.T. Peña, F. Gross, Eur. Phys. J. A 36, 329 (2008)

38. G. Ramalho, M.T. Peña, F. Gross, Phys. Rev. D 78, 114017 (2008)

39. G. Ramalho, K. Tsushima, Phys. Rev. D 81, 074020 (2010)

40. G. Ramalho, M.T. Peña, F. Gross, Phys. Lett. B 687, 335 (2009)

41. G. Ramalho, M.T. Peña, F. Gross, Phys. Rev. D 81, 113011 (2010)

42. F. Gross, J. Milana, Phys. Rev. D 43, 2401 (1991); 45, 969 (1992); 50, 3332 (1994)

43. C. Savkli, F. Gross, Phys. Rev. C 63, 035208 (2001)

44. F. Gross, K.M. Maung, Phys. Lett. B 229, 188 (1988)

45. F. Gross, K.M. Maung, Phys. Rev. C 42, 1681 (1990)

46. F. Gross, K.M. Maung, J.A. Tjon, L.W. Townsend, S.J. Wallace, Phys. Rev. C 40, R10 (1989)

47. K.M. Maung, L.W. Townsend, F. Gross, J.A. Tjon, S.J. Wallace, Phys. Rev. C 43, 1378 (1991)

48. F. Gross, S. Liuti, Phys. Rev. C 45, 1374 (1992)

49. S. Liuti, F. Gross, Phys. Lett. B 356, 157 (1995) 